\title{
Tungsten Thermionic Emission as a Gauge for Low Pressures of Cesium Vapor
}

\author{
João Shida ${ }^{1, *} \mathbb{*}$, Fangjian Wu ${ }^{1}\left(\mathbb{C}\right.$, Eric Spieglan ${ }^{1}$ and Mesut Çalışkan ${ }^{2} \mathbb{C}$ \\ 1 Enrico Fermi Institute, the University of Chicago, Chicago, IL 60637, USA; fjwu@uchicago.edu (F.W.); \\ eric.spieglan@gmail.com (E.S.) \\ 2 Kavli Institute for Cosmological Physics, the University of Chicago, Chicago, IL 60637, USA; \\ mesut@uchicago.edu \\ * Correspondence: joaofshida@uchicago.edu; Tel.: +1-312-833-3119
}

Received: 10 October 2020; Accepted: 2 November 2020; Published: 5 November 2020

\begin{abstract}
Heated metal filaments under electric fields and low pressures of alkali metal gas eject electrons by thermionic emission as a function of the pressure of the gas and the temperature of the filament. To explore this process in a program to develop large-area alkali metal photocathodes, we have designed and built a gauge following the studies of Taylor and Langmuir. We present proof-of-principle measurements of the thermionic emission of a tungsten filament in cesium vapor. We describe a second-generation design that corrects flaws in the first gauge.
\end{abstract}

Keywords: thermionic emission; cesium pressure gauge; tungsten work-function

\section{Introduction}

Alkali metals adsorb onto metal surfaces, with a concentration and structure dependent on the temperature of the surface and the pressure of the metal vapor. The adsorption increases the thermionic emission of the surface compared to bare metal. We show that, by measuring the thermionic emission as a function of the temperature of a tungsten filament, one can infer the pressure of cesium vapor around the filament.

According to Richardson's law, the thermionic current is solely determined by the work function of the surface and its temperature:

$$
J=\lambda_{R} A_{0} T^{2} e^{-W / k_{B} T}
$$

The work function changes with the coverage of adsorbed cesium atoms. For coverages between 0 and 0.66 , the adsorbed atoms lose an electron, creating an ionic charge layer which diminishes the work function and increases thermionic emission [1].

For coverages above 0.66 , the work function is low enough so that cesium ions that adsorb do not lose an electron, creating a neutral layer. To accommodate the larger radius of the neutral atoms, this layer is rotated $30^{\circ}$ about the normal [2] relative to the ionized layer below. The work function approaches that of cesium metal, suppressing thermionic emission.

Taylor and Langmuir [3,4] describe a relationship between the temperature of a tungsten filament, the cesium vapor pressure, and tungsten thermionic emission. The dotted curves in Figure 1 show the relationship of the thermionic emission as the temperature of the filament changes for different cesium atom fluxes, and is reproduced from Figure 15 in their 1933 paper [3]. 


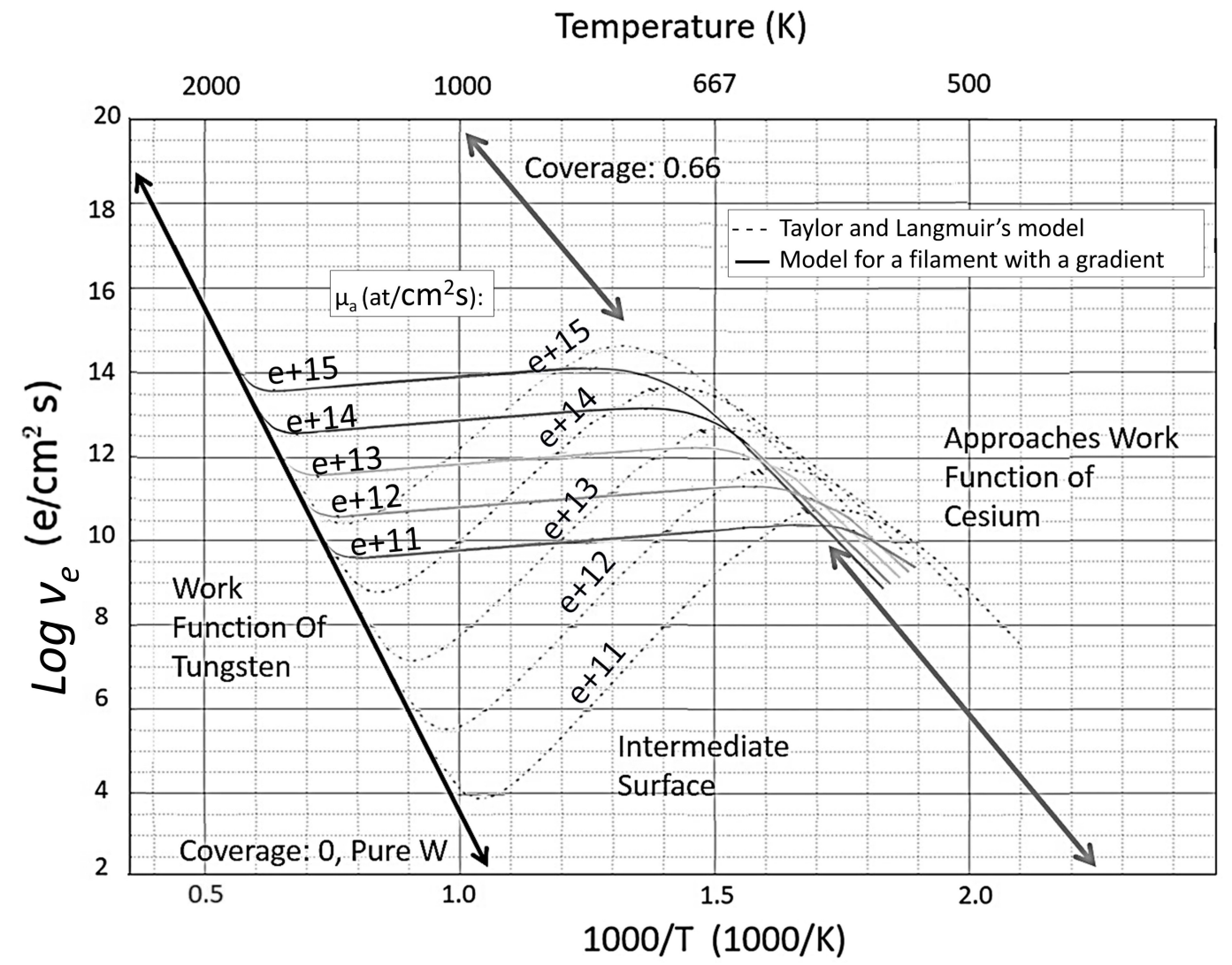

Figure 1. The electronic thermionic emission calculated with Taylor and Langmuir's measurements is shown as dotted lines for five values of cesium atom flux $\left(\mu_{a}\right)$; each curve is labeled with the atomic flux, where e+11 $\equiv 1 \times 10^{11}\left(\mathrm{at} / \mathrm{cm}^{2} \mathrm{~s}\right)$. To account for the thermal gradient of the filament in our gauge, we have corrected the Taylor and Langmuir model by integrating over the filament, with the result shown as solid lines. The features of the curves such as the peaks and valleys become flattened with a filament that has a temperature gradient: The hotter central parts of the filament emit orders of magnitude fewer electrons than the colder edges of the filament, which obscures the emission of the center and flattens the valley of the curve. The $y$-axis indicating thermionic emission is in the log scale.

In a program to develop large-area alkali metal photocathodes, we have explored thermionic emission by building a gauge to measure the partial pressure of cesium [5]. A crucial step in the creation of $\mathrm{Cs}_{3} \mathrm{Sb}$ photocathodes is the transfer of cesium onto a $\mathrm{Sb}$ surface via evaporation from a source far from the surface, followed by deposition [6,7]. The gauge was designed to monitor the cesium pressure during process, between $10^{-5}$ and $10^{-3} \mathrm{~Pa}$, although it was ultimately not implemented.

Section 2 describes the gauge construction, assembly, and operation. The results are presented in the context of the Taylor and Langmuir model in Section 3. Section 4 describes lessons we have learned from this first generation gauge and describes specific solutions to the issues we encountered.

\section{Materials and Methods}

The proof-of-concept gauge is shown in Figure 2. It consists of a $4.8514 \mathrm{~cm}$ by $0.0762 \mathrm{~cm}$ by $0.0025 \mathrm{~cm}$ tungsten ribbon filament from Scientific Instrument Services (SIS) surrounded by a collector for the thermionic current. The collector comprises a flat base made with a ceramic rectangular plate overlaid by a flat copper plate, on which is mounted a semi-cylindrical copper sheet, both cut from stock sheet copper of $0.001^{\prime \prime}$ thickness. The tungsten ribbon is supported above the rectangular plate by isolating screws at each end which serve as terminals for the filament. 

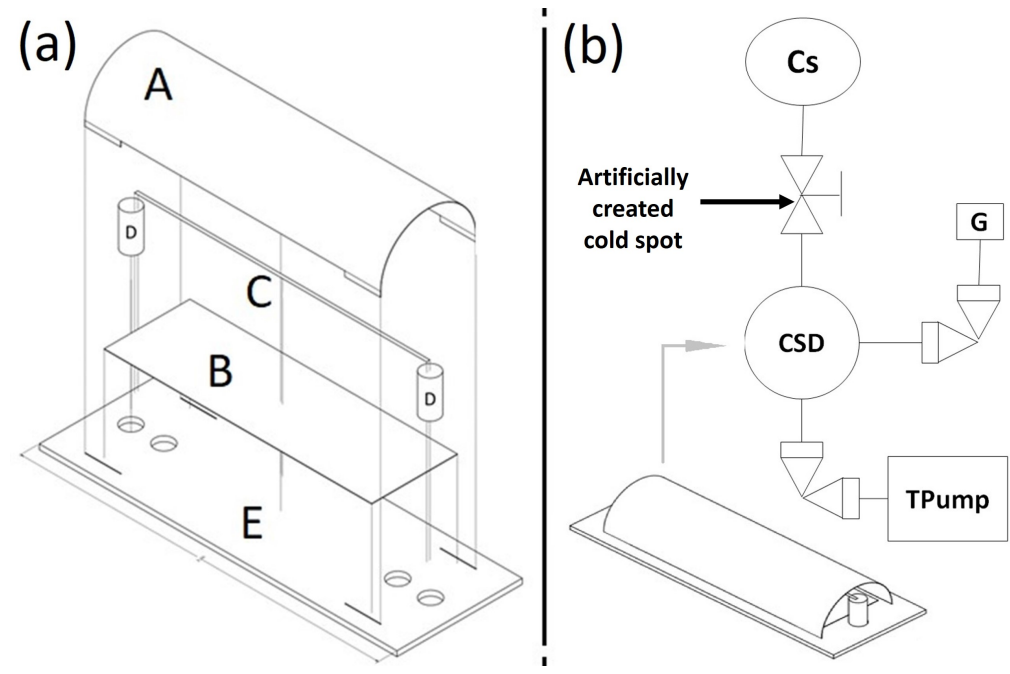

Figure 2. (a) On the left, the exploded assembly of the gauge. There are copper wires that were not depicted connecting the cylindrical (A) and flat (B) copper plates, as well as the filament ends, to independent pins in a feedthrough. The filament (C) is connected to screws which act as leads (D). All elements are isolated from each other through ceramic beads and boards like the base ceramic support (E). (b) On the right, a scheme of the manifold assembly. Label CSD indicates the cross chamber where the gauge is located, $\mathbf{C s}$ indicates the cesium source chamber, TPump is the turbopump and $\mathbf{G}$ is a pressure gauge. The copper wires connecting the gauge to the feedthrough are thick enough to support the structure without it dipping and touching the manifold. The cold spot is located $1 \mathrm{ft}$ away from the Gauge.

All components are inside a $23 / 4^{\prime \prime}$ CF 4-way cross flange, and are individually connected by ceramic-isolated copper wires to a vacuum feedthrough at one end of the cross. The cross chamber connects to an SS4-BG-V51 Swagelok valve leading to a custom cesium source containing a glass vial of $1 \mathrm{~g}$ of $99.9 \%$ cesium from ESPI Metals. The cross chamber is also connected to a HiPace 30 turbo-molecular pump from Pfeiffer Vacuum and a Compact FullRange ${ }^{\mathrm{TM}}$ Gauge PKR 251 gauge from Pfeiffer Vacuum through a valve [8]. The CF manifold pieces were DN40CF flanges and were metal-sealed, and all valves but the one to the cesium source were all-metal 541 angle valves from VMT.

To begin data-taking, the system was pumped to a pressure of $1.3 \times 10^{-5} \mathrm{~Pa}$, the FullRange ${ }^{\mathrm{TM}}$ gauge was valved off and the glass vial containing the cesium metal was broken to release cesium inside the cesium source chamber [6]. The turbo-molecular pump was valved off during measurements, but allowed to pump otherwise to remove hydrogen outgassing.

To achieve specific partial pressures of cesium, the manifold was heated, its temperature measured by 10 type $\mathrm{K}$ thermocouples and the temperature of a deliberately created cold spot recorded. The partial pressure of cesium was assumed to equilibrate after $12 \mathrm{~h}$ with the saturation pressure of the vapor over the cold spot. The manifold was heated by means of external glass-fiber insulated heat cable under a jacket of fiberglass insulation and the cold spot was created by omitting insulation from part of the external surface.

The circuit used to measure thermionic emission is outlined in Figure 3. It comprises two subsystems, the heating of the filament, and collectors that measure thermionic emission. The heating circuit consists of a power supply with a built-in ammeter and voltmeter (A and V in Figure 3) connected to the screws at the ends of the tungsten filament, with the negative terminal set at manifold ground. The measurement circuit consists of the copper plate and semi-circular sheet collectors tied to a bias voltage relative to ground through a $10.03 \mathrm{k} \Omega$ resistor. The voltage from the collector current, typically $0.01-100 \mathrm{~V}$, is measured across this resistor. 


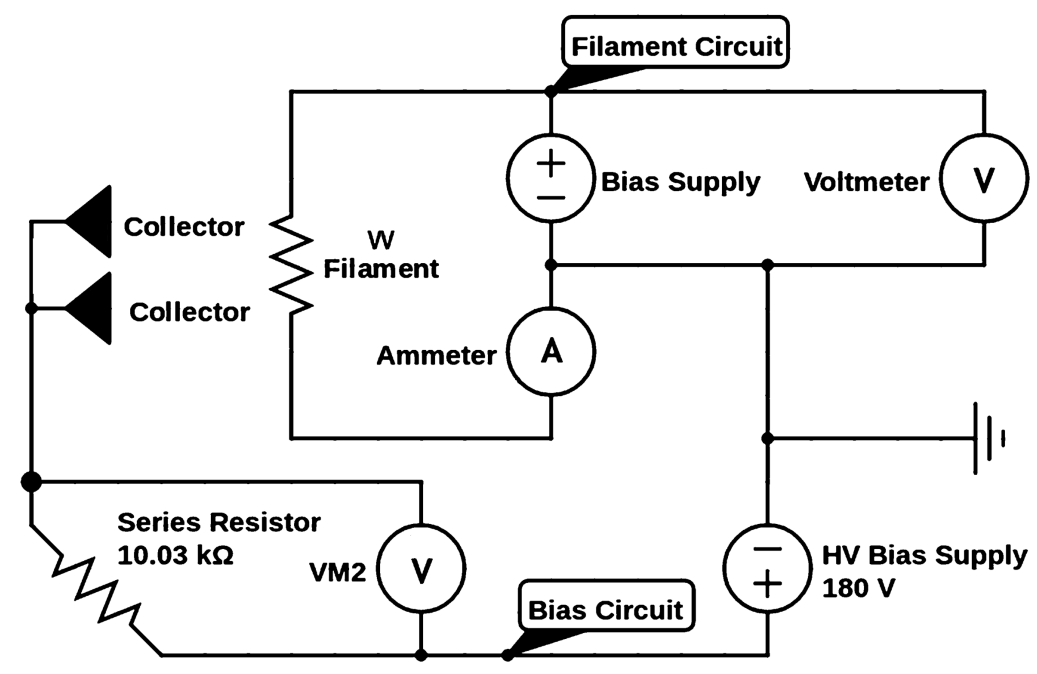

Figure 3. Circuitry of the gauge. Thermionic electrons leave the filament at ground voltage and are pulled by an electric field towards the collectors at a high voltage $(>200 \mathrm{~V}+)$. They then pass through the series resistor and the voltage in voltmeter 2 is proportional to the thermionic emission from the filament.

As the bias voltage increases from zero, more thermionic electrons are directed to the collectors. There is a critical bias voltage after which the current plateaus because all emitted electrons reach the filament. We reproduced Figure 21 in the Taylor and Langmuir paper [3] to find this point: 180-200 V as shown in Figure 4. All measurements were performed at or beyond this critical bias voltage.

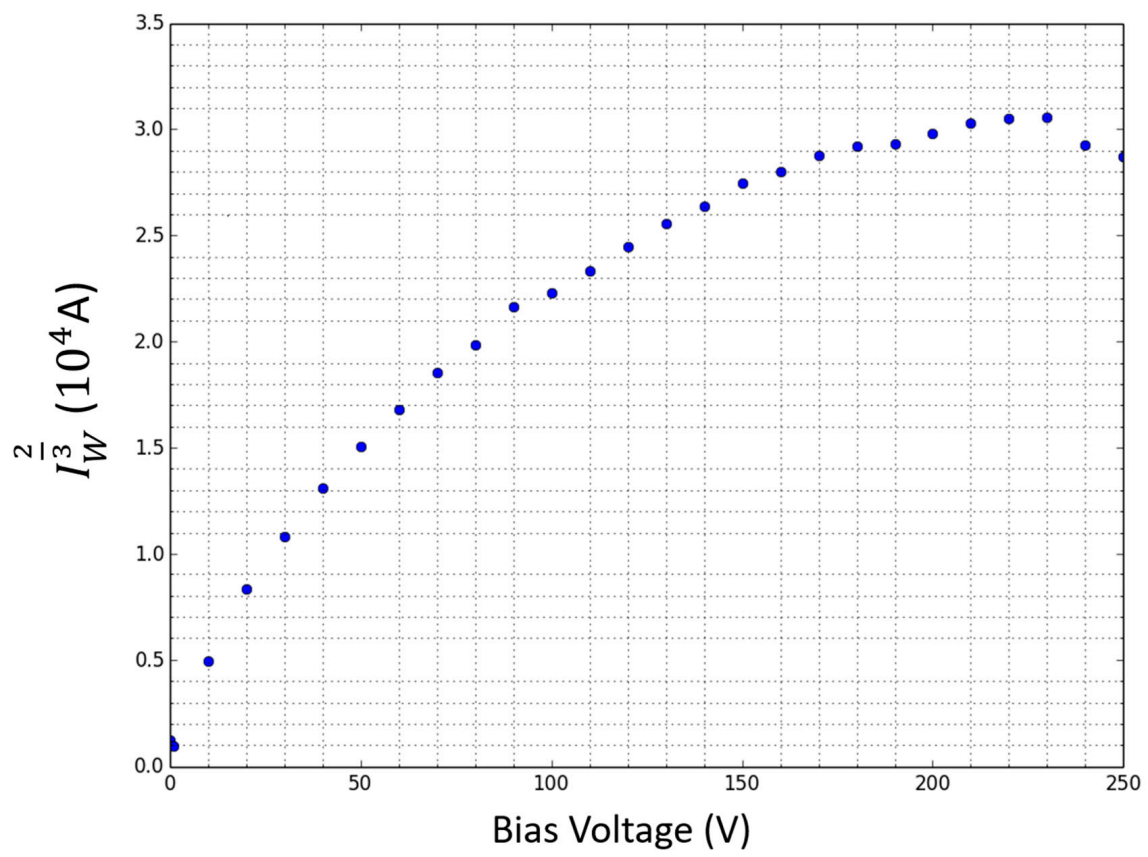

Figure 4. Curve of measured thermionic current versus increased bias voltage (difference between collectors and filament). In this case, the critical voltage observed was around $200 \mathrm{~V}$ and, after that, the collected current started plateauing. If measurements were taken at a voltage before the plateau, not all electrons would be collected for some temperatures of the filament, which would indicate a lower pressure. The current was manipulated as the $y$-axis indicates to be in the same form as Figure 21 in the Taylor and Langmuir paper [3]. 
A measurement with the gauge consists of changing the filament temperature by changing the current and measuring the collection current by the voltage through the $10.03 \mathrm{k} \Omega$ resistor. The collected electron current divided by the surface area of the filament yields the thermionic electron flux. The conversion between filament current to filament temperature invokes a temperature transport equation, as described in Appendix A.

The measurements can be fit to the model of Taylor and Langmuir, which is used to infer the flux of cesium atoms in the manifold, and hence the Cs partial pressure. Measurements were taken without the aid of automated logging using voltmeters with $0.01 \mathrm{~V}$ precision. An automated measurement algorithm could easily be implemented.

Before each measurement, the circuit was probed for shorts between circuit elements and/or ground from cesium condensation on the gauge surfaces. To eliminate the shorts, we applied $300 \mathrm{~V}$ between each element and between the elements and ground. The initial low resistances increased to over $10 \mathrm{k} \Omega$ once the bias was applied. If the system was left idle for more than two hours, even if heated, the shorts returned and the process had to be repeated.

\section{Results}

Measurements of thermionic emission were taken at source temperatures of $363 \mathrm{~K}$ (Measurement A) and $305 \mathrm{~K}$ (Measurement B) with the manifold temperatures at $503 \mathrm{~K}$ and $516 \mathrm{~K}$, respectively. The measured pressures fit to $1.30 \times 10^{-4} \mathrm{~Pa}$ and $8.34 \times 10^{-4} \mathrm{~Pa}$ as shown in Figure 5. As expected, thermionic emission is greater at higher source temperatures. The results match a pure tungsten curve at high filament temperatures.

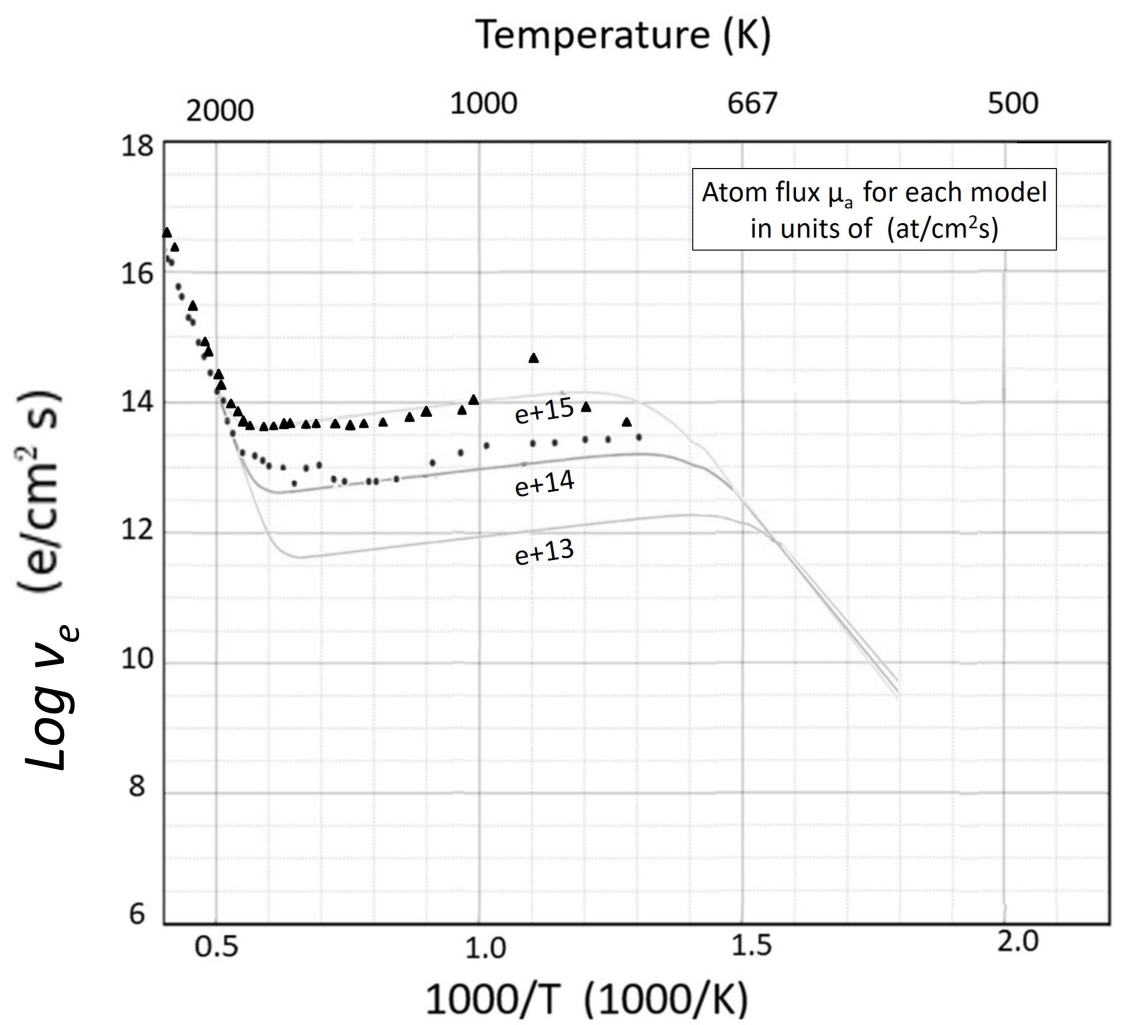

Figure 5. The logarithm of thermionic emission as measured by the gauge versus 1000/Filament Temperature for two measurements. Measurement A (triangles) was taken at a higher partial pressure of cesium than measurement B (circles), with the cesium source temperatures being $363 \mathrm{~K}$ and $305 \mathrm{~K}$, respectively. The outlier in measurement $\mathrm{A}$ is most likely a mistake. Each curve is labeled with the atomic flux, where $+13 \equiv 1 \times 10^{13}\left(\mathrm{at} / \mathrm{cm}^{2} \mathrm{~s}\right)$. 
The measured data do not match Taylor and Langmuir's (TL) predictions. The filament we used had a temperature gradient due to thermal conduction at the leads. In contrast, the TL results were obtained by using guard rings to suppress collection from filament segments that were not uniform in temperature: electronic thermionic emission was nearly uniform along the fraction of the filament measured. A correction based on the temperature gradient was computed, described in Appendix A. The corrected curve can be seen in Figure 1 as the solid lines, and in Figures 5 and 6. The corrected model agrees much better with the data.

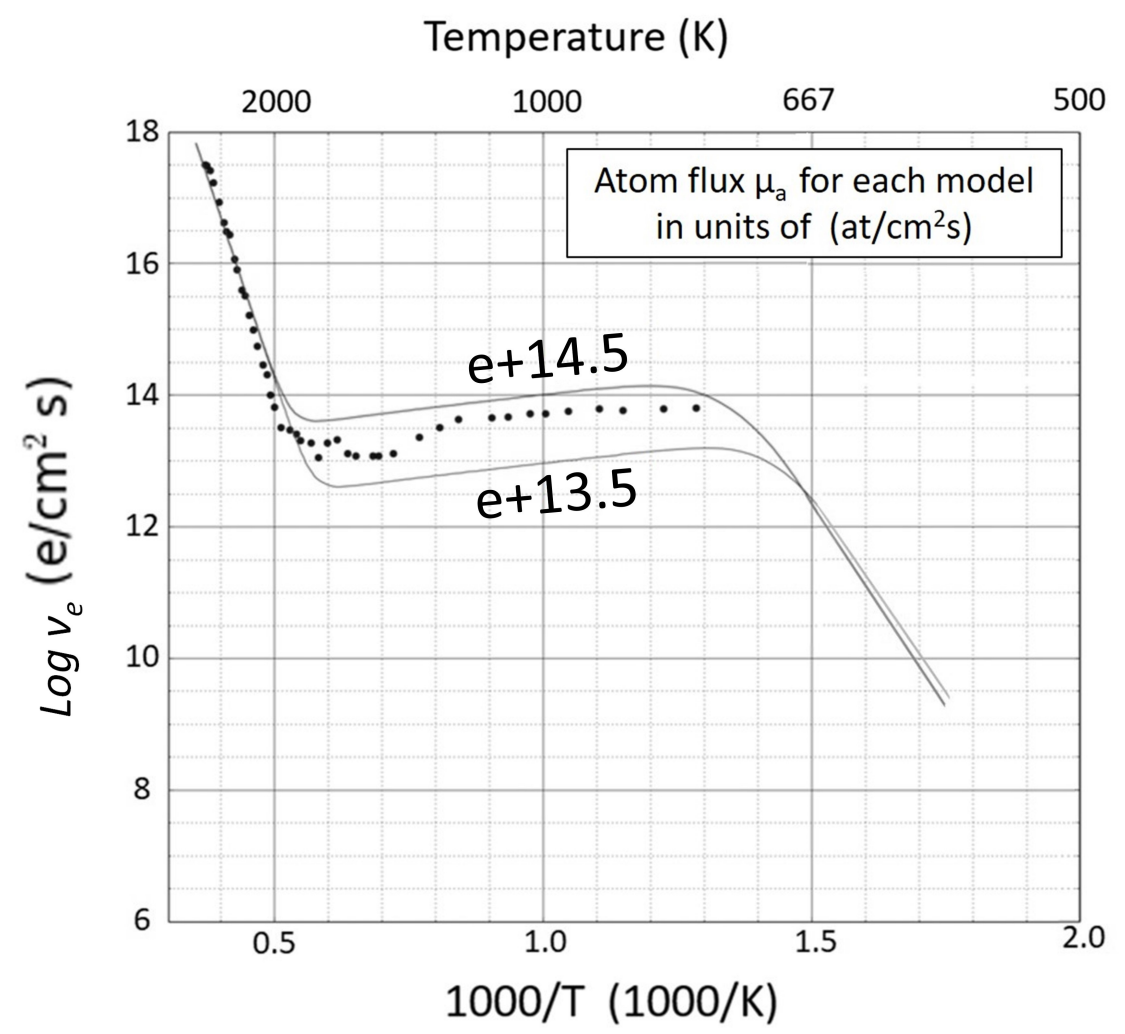

Figure 6. One measurement of the thermionic emission curve as a function of temperature. This measurement is fit to a solid curve corresponding to $10^{14.23} \mathrm{at} / \mathrm{cm}^{2} \mathrm{~s}$, an atom flux which can be converted to $1.30 \times 10^{-4} \mathrm{~Pa}$. The $y$-axis is in Log scale and each curve is labeled with the atomic flux, where $\mathrm{e}+14 \equiv 1 \times 10^{14}\left(\mathrm{at} / \mathrm{cm}^{2} \mathrm{~s}\right)$.

As filament temperatures decrease towards that of the manifold, heat conduction into the filament becomes significant, altering the relationship between filament current and temperature. The gauge was operated at elevated temperatures $\left(>200{ }^{\circ} \mathrm{C}\right.$, above $500 \mathrm{~K}$ for the measurements) to minimize cesium condensation; results at filament temperatures close to that of the manifold are omitted.

The proof-of-principle gauge failed after commissioning and two series of measurements due to cesium condensation impervious to evaporation. After the system had been opened and exposed to air, we verified a darkening of essentially all ceramic surfaces. Between electronic terminals, lighter patches appeared. This coloration pattern can be explained by cesium deposition and later vaporization between electronic terminals. When exposed to air, the remaining cesium darkened. For a more in-depth discussion on operation precautions and leakage currents, see Appendix B.

\section{Discussion: Proposed Second Generation Gauge}

The proof-of-concept gauge demonstrated the Taylor and Langmuir model can be used to measure cesium pressures, but made apparent that we had made mistakes in the design. The condensation of cesium vapor shorting circuit elements, and the presence of a filament temperature gradient are 
the main issues. An example second-generation instrument is illustrated in Figure 7 and described in Section 4.1. Alternatively, Springer and Cameron had proposed another method using a Bayard-Alpert gauge (BAG) to measure partial pressures of cesium using its ions instead of electrons $[9,10]$, as described in Section 4.2.

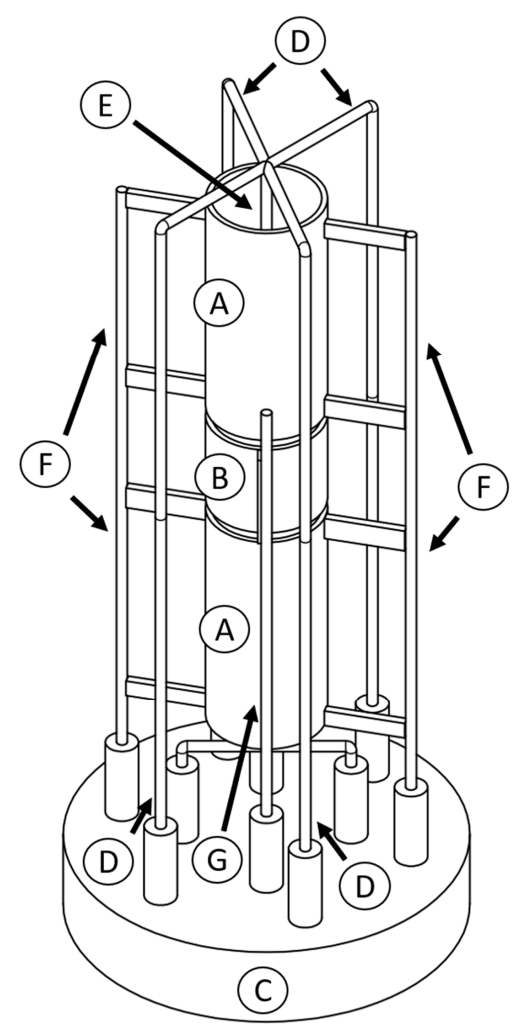

Figure 7. A scheme for a possible gauge redesign based on the results of the proof-of-concept gauge and its shortcomings. This design includes guard rings and minimizes structural-nonconducting surfaces to avoid leakage currents from cesium deposition. (A) indicates the guard-rings which produce no measurements. They are held up by structural wires indicated by (F). (B) indicates the central measurement ring, held up and isolated from the other elements by the structural wires indicated by (G). The cylindrical filament is indicated by (E) and is held taught by structural wires indicated by (D). All structural wires are connected to a feedthrough indicated by $(\mathrm{C})$ using ceramic connectors.

\subsection{Improved Gauge Using Thermionic Emission}

In order to counter leakage currents and electrical shorts, the gauge must minimize areas for cesium deposition around relevant conductors such as the collector or the leads. All circuit elements must avoid contact with support structures if possible. One solution is to have the circuit elements be self-supporting as in Figure 7.

To account for the filament temperature gradient, the collector may be split into two guard rings and a central collector ring (Figures 7 and 8). All rings should be cylindrical and biased at the same voltage to create a uniform radial electric field. Only the central guard ring would be used to measure thermionic emission as the center is the hottest part of the filament with the smallest gradients. This was done by Taylor and Langmuir [3].

The effectiveness of the guard rings is improved if the ends of the filament are coiled, increasing the length of the filament and thus flattening the temperature gradient. Although the emissions from the coiled areas will not follow the Taylor and Langmuir model, they will be shielded by the guard rings and not measured by the central ring. 


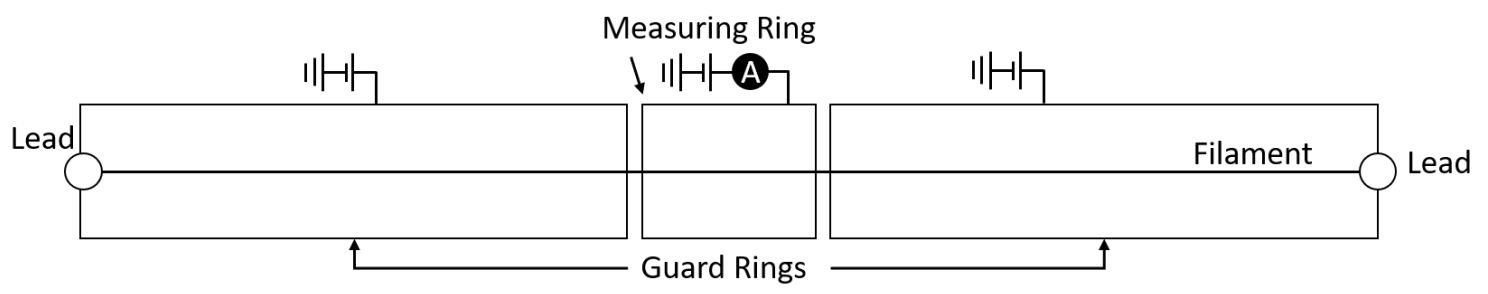

Figure 8. A scheme for how guard rings and the measurement conductor would be arranged. All three conductors would be biased at the same voltage and have the same shape. Around the center of the filament, the temperature can be considered almost constant. This means that, instead of a flattened curve, we would measure the same curve Taylor and Langmuir predict. Cylinders attached to the base are made of ceramic/insulating material, but all other elements are metal. It is paramount that the setup is structurally rigid to avoid shorts from small mechanical impacts/deformations.

\subsection{Using Ionized Cesium over Electrons as an Alternative}

An alternative method is to measure the current of ionized cesium ions instead of electrons thermionically emitted from the metal surface. A commercial Bayard-Alpert gauge (BAG) is an ion gauge with an emitter filament, a collector filament, and a cylindrical grid along the collector length. Springer and Cameron heated the grid to eject electrons and ionize cesium and biased the collector filament negatively to collect the ions. Measuring the collected current yields the cesium partial pressure in a similar way to the method described in this paper.

We had originally intended for the proof-of-principle gauge to be usable in ionic and electronic regimes. However, given the difficulties with cesium condensation, the electron regime was preferred. A description of a different ionization-based gauge and an additional method based on spectroscopic techniques are documented by Pradel, Roussel, and Spiess [11].

\section{Conclusions}

From the relationship between the thermionic emission of a tungsten filament and its temperature in the presence of cesium vapor, a gauge can be built to determine the partial pressure of the cesium. We have shown a proof-of-concept gauge and measurements, documented its problems, and proposed a new design. We hope this paper is useful to those who wish to construct a similar device or to explore the principles of thermionic emission, metallic crystal growth on metal surfaces, and work-functions.

Author Contributions: Conceptualization, F.W., E.S. and M.Ç.; Data curation, F.W.; Formal analysis, J.S.; Investigation, J.S., F.W. and E.S.; Methodology, J.S., F.W. and E.S.; Resources, E.S.; Software, J.S.; Supervision, E.S.; Visualization, J.S.; Writing—original draft, J.S.; Writing—review \& editing, J.S., F.W., E.S. and M.Ç. All authors have read and agreed to the published version of the manuscript.

Funding: This research received no external funding.

Acknowledgments: The authors would like to thank Evan Angelico, Andrey Elagin, Henry Frisch, and Mary Heintz for their help during the project; Matthew Poelker and Charles Sinclair for their insightful comments and helpful suggestions. We also thank Nicole Dombrowski, Hayward Melton, and Hannah Tomlinson for their previous work on assembling the proof-of-concept gauge. M.Ç. is supported by the Enrico Fermi Institute.

Conflicts of Interest: The authors declare no conflict of interest.

\section{Appendix A. Calculations for Wire Temperature Gradient}

To obtain the partial pressure of cesium, we must produce an experimental curve of thermionic emission versus temperature of the filament. To do so, it is necessary to convert the two observable parameters, the current through the tungsten filament $\left(I_{w}\right)$ and the current observed entering collecting conductors $\left(I_{c}\right)$ to the temperature of the filament $\left(T_{w}\right)$ and to the number of electrons leaving the filament $\left(v_{e}\right)$, respectively. 
The temperature of the filament is paramount to the characterization of the thermionic current. In their experiments, Taylor and Langmuir made use of guard rings to only measure thermionic electrons from the very center of their filaments so that they may safely assume that its temperature is uniform in that region. However, without using guard rings, and instead measuring electrons from the entire length of the filament, it is necessary to account for the filament's temperature gradient.

Since the leads conduct heat away from the filament, there is a temperature gradient with a peak at the midpoint. The equation that represents the equilibrium (a steady temperature distribution) for a small part of the filament is:

$$
0=d P e+d P t i-d P i-d P t o
$$

Where $d P e$ is the power lost by emission, $d P i$ is the power from the current through the filament, $d P t i$ is the power conducted in from the hotter end and $d P$ to is the power conducted out at the colder end (see Figure A1). Each of these components can be written out as a function of the position in the filament as follows:

$$
\begin{gathered}
d P e=d A_{e} \epsilon_{\left(T_{(x)}\right)} \sigma T_{(x)}^{4} \\
d P i=\frac{i^{2} \rho_{\left(T_{(x)}\right)} d x}{A_{t}} \\
d P t i=A_{t} K_{\left(T_{\left(x_{3}\right)}\right)} \frac{T_{\left(x_{4}\right)}-T_{(x)}}{d x} \\
d P t o=A_{t} K_{\left(T_{\left(x_{2}\right)}\right)} \frac{T_{(x)}-T_{\left(x_{1}\right)}}{d x}
\end{gathered}
$$

where $l, w$ and $d$ are the dimensions of the filament, with $l$ being its long edge, $A_{e}$ is the external area of the filament, $A_{t}$ is the transverse area of the filament (in our case of a flat filament, $A_{e}=2 l(w+d)$ and $A_{t}=w d$, respectively); $\sigma$ is the Stefan-Boltzmann constant, $T_{(x)}$ is the temperature gradient; $i$ is the current through the filament. Furthermore, $\rho_{\left(T_{(x)}\right)}$ is the resistivity of tungsten as a function of temperature; $\epsilon_{\left(T_{(x)}\right)}$ is the emissivity of tungsten as a function of temperature; and $K_{\left(T_{(x)}\right)}$ is the conductivity of tungsten as a function of temperature. These three tungsten properties were all fitted to 4 th or 5 th order polynomials as a function of $\mathrm{T}$.

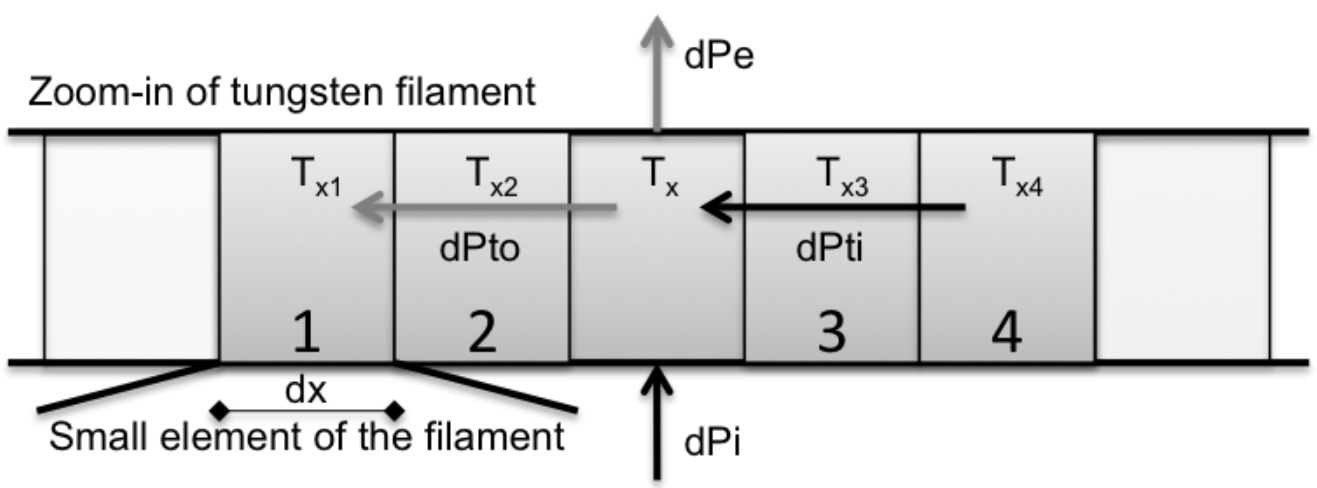

Figure A1. Power transport scheme for a small piece of the filament, the different pieces are numbered as they are used in Equations (A2)-(A9). The Black arrows indicate power into the filament piece, and the grey arrows indicate power out. $d P t i$ is the power piece 3 conducts from piece 4 into the center piece, and $d P$ to is the power piece 2 that conducts from the center piece to piece 1 .

Note that Equations (A4) and (A5) can be re-written as a function of the first derivative of $T_{(x)}$ at $x_{3}$ and $x_{2}$, respectively:

$$
d P t i=A_{t} K_{\left(T_{\left(x_{3}\right)}\right)} \frac{d T\left(x_{3}\right)}{d x}
$$




$$
d P \text { to }=A_{t} K_{\left(T_{\left(x_{2}\right)}\right)} \frac{d T\left(x_{2}\right)}{d x}
$$

Substituting Equations (A2), (A3), (A6), and (A7) back into Equation (A1), we have:

$$
0=d A_{e} \epsilon_{\left(T_{(x)}\right)} \sigma T_{(x)}^{4}+\frac{i^{2} \rho_{\left(T_{(x)}\right)} d x}{A_{t}}+\left(A_{t} K_{\left(T_{\left(x_{3}\right)}\right)} \frac{d T\left(x_{3}\right)}{d x}-A_{t} K_{\left(T_{\left(x_{2}\right)}\right)} \frac{d T\left(x_{2}\right)}{d x}\right)
$$

which can also be re-written as a function of the second derivative of $T_{(x)}$ :

$$
0=d A_{e} \epsilon_{\left(T_{(x)}\right)} \sigma T_{(x)}^{4}+\frac{i^{2} \rho_{\left(T_{(x)}\right)} d x}{A_{t}}+A_{t}\left(\left(\frac{d T_{(x)}}{d x}\right)^{2} \frac{d K_{\left(T_{(x)}\right)}}{d T_{(x)}}+K_{\left(T_{(x)}\right)} \frac{d T_{(x)}^{2}}{d^{2} x}\right) d x
$$

This is a second order differential equation that can be solved numerically for $T_{x}$ with two boundary conditions. We assume knowledge of the temperature of the ends of the filament, which is valid if the leads they are connected to are heat-sunk to the manifold. The manifold temperature is measurable from the outside. Furthermore, given that at high temperatures $K_{(T)}$ is small, we assume that, for the very center of the wire, where it is hottest, Equation (A1) has negligible conduction summands, and thus we can solve for the temperature at the midpoint given a current. Therefore, we have both $T_{(0)}$ and $T_{\left(\frac{l}{2}\right)}$ as boundary conditions. Solving for $T_{(x)}$, we get, for any current, a temperature gradient in the filament.

Once we have $T_{(x)}$, we can perform a numerical integration of how many thermionic electrons will be emitted by different parts of the filament based on Taylor and Langmuir's experimentally determined curve. We do this to calculate the predicted thermionic emission from a filament with non uniform temperatures, and we can generate a curve analogous to Taylor and Langmuir's but for a filament with a temperature gradient instead of a uniformly heated filament (See dotted lines in Figure 1).

\section{Appendix B. Operation Precautions}

A measurement with the gauge has been described above. However, to obtain reproducible results, certain precautions must be taken.

Taylor and Langmuir recommend that before any measurement is done the filament undergoes what they call an aging process. This involves leaving the filament heated at $2400 \mathrm{~K}$ for $10 \mathrm{~h}$, then at $2600 \mathrm{~K}$ for an hour, and finally concludes with a few brief flashes at $2900 \mathrm{~K}$. Prior to such aging, neither we nor Taylor and Langmuir could obtain reproducible results. Furthermore, we have determined that brief flashes to about $2500 \mathrm{~K}$ before any measurement generate more reproducible results as well.

It is also necessary that the measurement not interfere appreciably with the temperature of the source of cesium. The filament heats up to high temperatures during the measurement (up to $2500 \mathrm{~K}$ ) and, if the cesium source temperature changes mid-measurement, so will the pressure of cesium vapor. A fast measurement can solve this issue, as well as placing the source far away from the filament or using a thinner filament.

Leakage currents between the charged plates and either ground or the filament terminals increase the background in the measurement of the currents collected from the plates. We attribute this leakage current to condensed cesium on the ceramic tile connecting the ends of the filament, the plates, and the manifold together.

There are two ways leakage currents interfere with the measurements. It makes it so that the signal measured across the series resistor does not only come from collected thermionic electrons, but also from ground electrons that arrive from leakage conductive paths. Furthermore, as one changes the temperature of the conductive paths, cesium evaporates or condenses, thus changing the resistance of the leakage pathways. This means that there is not a simple static current background one might subtract from the measured signal. 
It is possible to deal with such currents. By applying high voltages to the circuit elements, any conductive cesium paths to ground will heat up and the cesium will evaporate off the surfaces. For the charged plates, half an hour at $300 \mathrm{~V}$ took a $10 \mathrm{k} \Omega$ short to up to tens of $\mathrm{M} \Omega$. Such voltages required the use of high-voltage power source. Another effective counter to leakage currents was to keep the gauge much hotter than the source to avoid creating sites prone to cesium deposition. When source and manifold were left at the about the same temperature, all of the circuit elements were connected to each other and to the ground by resistances under one $\mathrm{k} \Omega$. When the manifold was left at four times the temperature of the source, however, resistances went up to hundreds of $\mathrm{k} \Omega$.

\section{References}

1. Gavrilyuk, V.M.; Naumovets, A.G.; Fedorus, A.G. Investigation of adsorption of cesium on a tungsten single crystal. J. Exp. Theor. Phys. (U.S.S.R.) 1966, 51, 1332-1340.

2. Naumovets, A.G.; Fedorus, A.G. Cesium on tungsten (011) face; structure and work function. Surf. Sci. 1970, 21, 426-439

3. Taylor, J.B.; Langmuir, L.I. The Evaporation of Atoms, Ions and Electrons from Caesium Films on Tungsten. Phys. Rev. 1933, 44, 423. [CrossRef]

4. Taylor, J.B.; Langmuir, I. Vapor Pressure of Caesium by the Positive Ion Method. Phys. Rev. 1937, 51, 753. [CrossRef]

5. Sinclair, C. Alkali Metal Sources for Photocathodes and their Quantification-Getter Sources vs. Metallic Evaporation Source. In Proceedings of the UChicago Photocathode Workshop, Chicago, IL, USA, 29-30 June 2012.

6. Angelico, E.; Spieglan, E.; Elagin, A.; Frisch, H.; Bernhard, A. Dual Low Vacuum-UltraHigh Vacuum System for Large-Scale Production of Micro-Channel Plate Photomultipliers. U.S. Patent 62928598, 31 October 2019.

7. Angelico, E.; Elagin, A.; Frisch, H.; Spieglan, E.; Adams, B.; Foley, M.; Minot, M. Air-transfer production method for large-area picosecond photodetectors. Rev. Sci. 2020, 91, 053105. [CrossRef] [PubMed]

8. Pfeiffer Vaccum. Compact FullRange Gauge PKR 251 Instruction Manual; Pfeiffer Vaccum: Nashua, NH, USA, 2008.

9. Springer, R.W.; Cameron, B.J. Photocathode transfer and storage techniques using alkali vapor feedback control. In Proceedings of the 13th International Free-Electron Laser Conference, Santa Fe, NM, USA, 25-30 August 1991; Volume 92, p. 753.

10. Charles, S. Cornell University, Ithaca, NY, USA. Personal communication, 2020.

11. Pradel, P.; Roussel, F.; Spiess, G. Measurements of the vapor pressure of cesium by absorption of resonance radiation, $\lambda=8521 \AA$ A. Rev. Sci. Instrum. 1974, 45, 45-50. [CrossRef]

Publisher's Note: MDPI stays neutral with regard to jurisdictional claims in published maps and institutional affiliations.

(C) 2020 by the authors. Licensee MDPI, Basel, Switzerland. This article is an open access article distributed under the terms and conditions of the Creative Commons Attribution (CC BY) license (http:/ / creativecommons.org/licenses/by/4.0/). 